\title{
Antitumor activity of NRC-AN-019 in a pre-clinical breast cancer model
}

\author{
CHRISTOPHER S. GONDI ${ }^{1}$, BHARATHI GORANTLA ${ }^{1}$, A.K.S. BHUJANGA RAO ${ }^{3}$, K. AMALA ${ }^{3}$, M.U.R. NAIDU ${ }^{4}$, \\ K.V. JOGI ${ }^{3}$, G. VENKAT RAMANA ${ }^{3}$, PRAVEEN C. MYNENI ${ }^{3}$, AJIT JUNNARKAR ${ }^{3}$ and JASTI S. RAO ${ }^{1,2}$ \\ Departments of ${ }^{1}$ Cancer Biology and Pharmacology, and ${ }^{2}$ Neurosurgery, University of Illinois College of Medicine \\ at Peoria, Peoria, IL, USA; ${ }^{3}$ NATCO Pharma Limited; ${ }^{4}$ Nizam's Institute of Medical Sciences, Hyderabad, India
}

Received April 5, 2011; Accepted May 18, 2011

DOI: $10.3892 /$ ijo.2011.1079

\begin{abstract}
Breast cancer is the second most frequently diagnosed tumor in women. Overexpression of human epidermal growth factor receptors (EGFRs) represents a biological subclass of breast cancer with distinct molecular alterations, clinical behavior and response to systemic therapy. In this study, we describe a novel compound (NRC-AN-019), which has better antitumor activity than Lapatinib. Here, we demonstrate that NRC-AN-019 is more effective in inhibiting angiogenic potential and proliferation of both MDAMB231 and HTB20/BT474 cells. FACS analysis shows that NRC-AN019 treatment caused the accumulation of MDAMB231 and BT474 cells in the sub G0/1 phase in a dose-dependent manner and was accompanied by increased PARP cleavage, which is indicative of apoptosis. In addition, we observed inhibition of EGFR phosphorylation in both MDAMB231 and BT474 cells. From our animal studies using SCID mice implanted with BT474 cells, we observed dose-dependent inhibition of tumor growth in NRC-AN-019-treated animals compared to controls or Lapatinib-treated mice at comparable concentrations. The dose-dependent inhibition of EGFR phosphorylation was confirmed by immunohistochemical analysis of tumor sections. In vitro results demonstrate that NRC-AN-019 is superior to Lapatinib in EGFR-overexpressing cells and has strong anti-angiogenic, anti-proliferative and pro-apoptotic properties in an EGFR-overexpressing background (BT474). In vivo studies demonstrate that the antitumor activity of NRC-AN-019 is better over Lapatinib. These results suggest
\end{abstract}

Correspondence to: Dr J.S. Rao, Department of Cancer Biology and Pharmacology, University of Illinois College of Medicine at Peoria, One Illini Drive, Peoria, IL 61605, USA

E-mail: jsrao@uic.edu

Dr A.K.S. Bhujanga Rao, NATCO Research Centre, B-13, Industrial Estate, Sanath Nagar, Hyderabad 500018, India

E-mail:nrc@natcopharma.co.in

Key words: breast cancer, human epidermal growth factor receptor, Lapatinib, NRC-AN-019 that NRC-AN-019 has greater therapeutic potential in the treatment of Her-2-positive breast cancer.

\section{Introduction}

Recent studies have shown that breast cancer incidence in women in the United States is 1 in $8(\sim 13 \%)$. In 2008, an estimated 182,460 new cases of invasive breast cancer were diagnosed in women in the US, along with 67,770 new cases of non-invasive (in situ) breast cancer. Recent world-wide statistics have determined that in 2010, 1.5 million people worldwide will be diagnosed with breast cancer (1).

Targeted therapies with compounds that inhibit a specific target molecule represent a new perspective in the treatment of breast cancer. The ErbB family of receptor (EGFR, also called HER-1, ErbB-1), HER-2 (neu, ErbB-2), HER-3 (ErbB-3) and HER-4 (ErbB-4). All four receptors are tyrosine kinases and consist of an extracellular ligand-binding domain, a single membrane-spanning region, and intracellular tyrosine kinase and regulatory domains. The progression of malignant tumors is a consequence of multiple alterations of the genome such as Her-2. It is a protein with extracellular and intracellular domain with tyrosine kinase activity involved in signal transduction of cell growth and development. Amplification of the HER-2 receptor tyrosine kinase has been implicated in the pathogenesis of $25 \%$ of invasive human breast cancers. The aberrant signaling of HER-2 also contributes to tumor initiation and disease progression (2). Inhibition of HER-2, dramatically enhances the anti-tumor activity of chemotherapy (3).

The agents that are currently approved for HER-2-positive breast cancer include transtuzumb and Lapatinib, though, transtuzumab treatment is widely used for the treatment of HER-2-positive breast cancer, only $15 \%$ response as monotherapy and $49 \%$ as combination therapy have been achieved. On the other hand, lapatinib, a small molecule tyrosine kinase inhibitor is approved for the treatment of advanced or metastatic HER-2 positive breast cancer in combination with capecitabine.

In this study, we demonstrate the antitumor activity of NRC-AN-019, a small molecule tyrosine kinase inhibitor with dual activity on HER-2 and pEGFR expression. We have also made and attempt to compare the efficacy of the NRC-AN-019 with Lapatinib in in vitro and in vivo models with vehicle as a negative control. 


\section{Materials and methods}

Compounds. The test compound NRC-AN-019 was dissolved in DMSO (dimethyl sulfoxide) and used. DMSO was used as a vehicle control.

Cell and culture conditions. MDAMB231 and HTB20/BT474 cells were obtained from ATCC. MDAMB231 cells were cultured in Leibovitz's L-15 medium supplemented with $10 \%$ fetal bovine serum and cultured at $37^{\circ} \mathrm{C}$ in a $5 \% \mathrm{CO}_{2}$ humidified atmosphere. BT474 cells were cultured in ATCC complete 46-X Hybri-Care growth medium supplemented with $1.5 \mathrm{~g} / 1$ sodium bicarbonate and $10 \%$ fetal bovine serum at $37^{\circ} \mathrm{C}$ in a $5 \% \mathrm{CO}_{2}$ humidified atmosphere.

TUNEL assay and determination of $I C_{50}$ values. We determined the induction of apoptosis in breast cancer cells after we treated breast cancer cell lines MDAMB231 and BT474 with Lapatinib (0-317.9 $\mu \mathrm{M})$ or NRC-AN-019 $(0-579.7 \mu \mathrm{M})$. TUNEL assay was carried out using the APO-BrdU ${ }^{\text {Ti }}$ TUNEL Assay Kit (Invitrogen) as per the manufacturer's instructions. Percent apoptosis was determined by flow cytometry. $\mathrm{IC}_{50}$ values were determined according to a previously described protocol after seven days of drug treatment (4).

Angiogenic assay. We determined in vitro angiogenesis of MDAMB231 and BT474 breast cancer cells in the presence of specified concentrations of experimental compounds. Briefly, cells $\left(2 \times 10^{4} /\right.$ well $)$ were seeded in 8 -well chamber slides and treated with various concentrations of NRC-AN-019 and Lapatinib. After a 24-h incubation period, conditioned medium was removed and added to a $4 \times 10^{4}$ human dermal endothelial cell monolayer in 8-well chamber slides, and the human dermal endothelial cells were allowed to grow for $72 \mathrm{~h}$. Cells were then fixed in $3.7 \%$ formaldehyde and stained with $\mathrm{H} \& \mathrm{E}$ and photographed. Angiogenesis was quantified as percent of controls based on the formula modified from a previously described method:

$$
\frac{\sum_{a}(B x \times N x)}{\sum_{a}(B c \times N c)} \times 100
$$

where $\mathrm{Bc}$ is the number of branch points, $\mathrm{Nc}$ is the number of branches around a branch point of control, Bx is the number of branch points, Nx is the number of branches around a branch point of treatments, and a is the number of branch points counted, which is equal in both control and treated groups. Five replications were done to obtain statistically significant results where $\mathrm{a}=50$ which represents the number of branch points counted (5).

MTT proliferation assay. We determined the change in cell proliferation of breast cancer cells after we treated breast cancer cell lines MDAMB231 and BT474 with Lapatinib $(0-211.9 \mu \mathrm{M})$ or NRC-AN-019 $(0-386.5 \mu \mathrm{M})$. MTT assay was performed in a 96-well plate as per standard protocol. Briefly, 10,000 cells in $200 \mu \mathrm{l}$ media per well were plated in a 96-well plate and incubated overnight at $37^{\circ} \mathrm{C}$ in $5 \% \mathrm{CO}^{2}$ to allow for cell attachment. Vehicle or NRC-AN-019 $(0-19.3 \mu \mathrm{M})$ or Lapatinib (0-10.5 $\mu \mathrm{M})$ dissolved in DMSO were added to each plate $(2 \mu \mathrm{l} /$ well max $)$ and placed on a shaking table at $150 \mathrm{rpm}$ for $5 \mathrm{~min}$ for thorough mixing. The plate was further incubated at $37^{\circ} \mathrm{C}$ in $5 \% \mathrm{CO}_{2}$ for $72 \mathrm{~h}$ to allow the drug to take effect. A $5 \mathrm{mg} / \mathrm{ml}$ of MTT solution in PBS was made fresh, and $20 \mu \mathrm{l}$ of MTT solution were added to each well and placed on a shaking table (150 rpm) for $5 \mathrm{~min}$ to thoroughly mix the MTT into the media. The plate was further incubated at $37^{\circ} \mathrm{C}$ in $5 \% \mathrm{CO}_{2}$ for $1 \mathrm{~h}$ to allow the MTT to be metabolized after which excess media were discarded and the formazan precipitate resuspended in $200 \mu 1 \mathrm{DMSO}$. The precipitate was allowed to dissolve in DMSO completely and optical density at $560 \mathrm{~nm}$ was measured and subtracted from background at $670 \mathrm{~nm}$.

Antibodies. Antibodies were obtained from Abcam (Cambridge, MA). Cleaved PARP antibody (cat. no. ab4830) specifically recognizes the $85-\mathrm{kDa}$ fragment of cleaved PARP. Ki-67 is a cell cycle-related nuclear protein expressed by proliferating cells in all phases of the active cell cycle (G1, S, G2 and M phase) but is absent in $\mathrm{G} 0$ cells, and hence is an indicator of proliferation (cat. no. ab15580). EGFR (cat. no. ab2430) and pEGFR (cat. no. ab40815) overexpression is indicative of proliferating tumor cells and poor prognosis. GAPDH antibody was used to verify loading controls (cat. no. ab8245).

Western blot analysis. We carried out Western blot analysis of MDAMB231 and BT474 breast cancer cells in the presence of specified concentrations of compounds as per standard protocols. Cells were treated with vehicle or NRC-AN-019 or Lapatinib at the specified concentrations. Twenty-four hours after treatment, cells were collected and cell lysates extracted. Equal quantities of proteins $(100 \mu \mathrm{g})$ were fractionated by SDS-PAGE. The fractionated proteins were blotted onto nylon membranes and immunoprobed for cleaved PARP, Ki-67, EGFR and pEGFR as per standard protocols. The membranes were stripped and levels of GAPDH were determined; GAPDH served as a loading control.

Cell cycle analysis. To determine the change in cell cycle progression of breast cancer cells when treated with NRC-AN019 or Lapatinib, we treated breast cancer cell lines MDAMB231 and BT474 with Lapatinib $(0-105.9 \mu \mathrm{M})$ or NRC-AN-019 $(0-193.2 \mu \mathrm{M})$ and cultured for $12 \mathrm{~h}$. Higher molar concentrations of NRC-AN-019 were used to maintain equal weight concentrations used. Cells were then trypsinized and treated with $50 \mu \mathrm{g} / \mathrm{ml}$ propidium iodide $+0.001 \%$ RNAse-A solution as per standard protocols and sorted on a fluorescence-activated cell sorter (10,000 cells sorted).

Quantification of pEGFR expression by ELISA. We performed ELISA as per manufacturer's instructions (Cell Signaling Pathscan ELISA kit no. 7250, Danvers, MA) to determine total pEGFR levels. Briefly, cells were lysed in $1 \mathrm{X}$ cell lysis buffer [20 mM Tris (pH 7.5), $150 \mathrm{mM} \mathrm{NaCl}, 1 \mathrm{mM}$ ethylene diamine tetraacetate (EDTA), $1 \mathrm{mM}$ ethylene glycol-bis(2-aminoethyl)$\mathrm{N}, \mathrm{N}, \mathrm{N}^{\prime}, \mathrm{N}^{\prime}$-tetraacetic acid (EGTA), $1 \%$ Triton X-100, $2.5 \mathrm{mM}$ sodium pyrophosphate, $1 \mathrm{mM} \beta$-glycerophosphate, $1 \mathrm{mM}$ $\mathrm{Na}_{3} \mathrm{VO}_{4}, 1 \mu \mathrm{g} / \mathrm{ml}$ leupeptin] followed by sonication. Each diluted cell lysate $(100 \mu \mathrm{l})$ was added to the appropriate well 
and incubated for $2 \mathrm{~h}$ at $37^{\circ} \mathrm{C}$. Then, the detection antibody was added for $1 \mathrm{~h}$ at $37^{\circ} \mathrm{C}$ followed by HRP-conjugated secondary antibody for $30 \mathrm{~min}$ at $37^{\circ} \mathrm{C}$. Detection was done by the addition of $100 \mu \mathrm{l}$ of TMB substrate to each well followed by the addition of $100 \mu \mathrm{l}$ of STOP solution. Absorbance was read at $450 \mathrm{~nm}$ within $30 \mathrm{~min}$ of STOP solution addition.

Animal studies. For this study, SCID mice were used (homozygous genotype: NOD.CB17-Prkdc^scid/NCrHsd, NOD.CB17/ JHliHsd-Prkd $c^{\wedge} s c i d$ ). We used 8 mice per group (4 female and 4 male). Animals were subcutaneously implanted with $2 \times 10^{6}$ BT474 cells. After tumors developed (2-3 mm diameter), drug treatment was initiated. For Lapatinib: 30 and $100 \mathrm{mg} / \mathrm{kg}$ (two times a day) for 21 days by oral gavage. For NRC-AN-019: 10,20 and $40 \mathrm{mg} / \mathrm{kg}$ once daily for 30 days by oral gavage. Periodic tumor volumes were determined. Thirty days after initiation of drug treatment, mice were sacrificed and tumors harvested. Tumor volumes were measured using the formula,

$$
\text { tumor volume }=\frac{\pi}{6}\left(1 \mathrm{x} \mathrm{s}^{2}\right)
$$

where $1=$ large diameter and $\mathrm{s}=$ small diameter of tumor. After termination of the experiment, subcutaneous tumors were harvested and processed for paraffin sectioning followed by immunohistochemistry for pEGFR expression. All animal procedures were approved by the University of Illinois College of Medicine at Peoria, Instructional Animal Care Committee.

Immunohistochemistry. Tumors resected from SCID mice treated with NRC-AN-019, Lapatinib or vehicle controls were processed for paraffin sectioning as per standard protocols. Sections were deparaffinized as per standard protocol followed by blocking in $1 \%$ BSA in PBS for $1 \mathrm{~h}$, and the sections were subsequently transferred to primary antibody pEGFR diluted in $1 \%$ BSA in PBS (1:500). Sections were allowed to incubate in the primary antibody solution for $2 \mathrm{~h}$ at $4^{\circ} \mathrm{C}$ in a humidified chamber, followed by washing in $1 \%$ BSA in PBS (three 5-min washes), and further followed by the addition of appropriate HRP-conjugated secondary antibody in $1 \%$ BSA in PBS. After secondary antibody addition and subsequent washing, the sections were allowed to incubate in HRP DAB substrate as per standard protocols to develop color. Transmitted light images were obtained after counterstaining with hematoxylin as per standard protocol to visualize the morphology of the sections. A control study was performed using a normal rabbit immunoglobulin fraction as the primary antibody, which served as the negative control. The expression levels of pEGFR were quantified by measuring DAB pixel density per unit area (DAB pixels per 10 fields using a 1024x1024 image) and graphically represented as percent expression.

\section{Results}

NRC-AN-019 induces apoptosis in Lapatinib-resistant BT474 breast cancer cells. It was observed that NRC-AN-019 efficiently induced apoptosis at concentrations as low as $1.9 \mu \mathrm{M}$ in MDAMB231 cells, and $96.6 \mu \mathrm{M}$ in BT474 cells. From the quantitative analysis of apoptotic induction in MDAMB231 and BT474 cells, we observed that apoptotic induction by NRC-AN019 in MDAMB231 was more effective than Lapatinib treatment

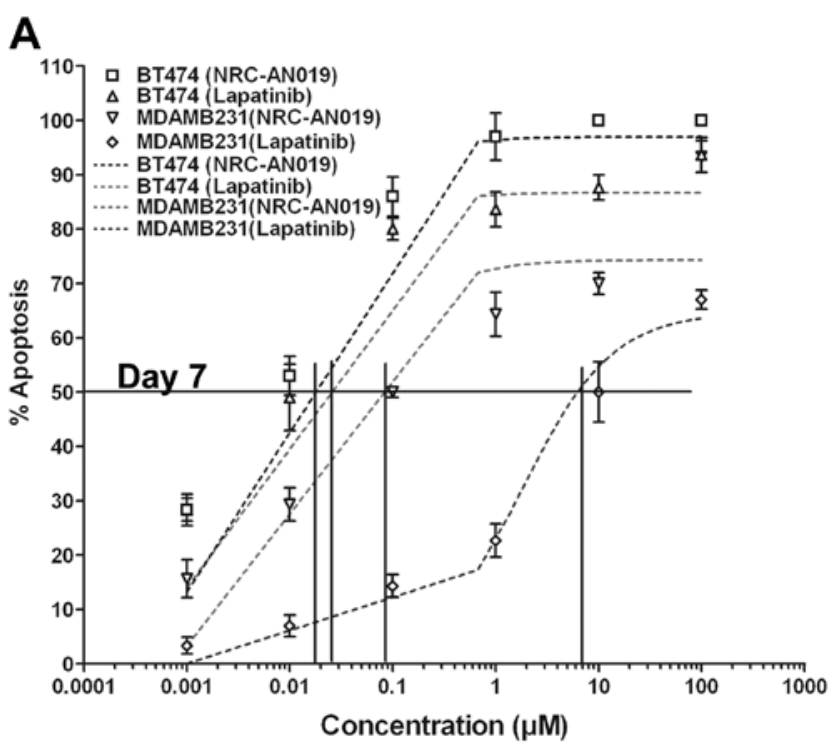

B

\begin{tabular}{c|cc} 
Cell lines & Compound & $I_{50}(\mu \mathrm{M})$ \\
\hline \multirow{2}{*}{ BT474 } & NRC-AN019 & $0.01^{\mathrm{a}}$ \\
& Lapatinib & $0.02^{\mathrm{b}}$ \\
MDAMB231 & NRC-AN019 & $0.85^{\mathrm{c}}$ \\
& Lapatinib & $7.01^{\mathrm{d}}$
\end{tabular}

Figure 1. NRC-AN-019 induces apoptosis in breast cancer cells. Human breast cancer cells MDAMB231 and BT474 were treated with varying concentrations of NRC-AN-019 or Lapatinib, followed by TUNEL assay using the APO-BrdU TUNEL assay kit (Invitrogen) as per manufacturer's instructions. Percent decrease in proliferation is graphically presented (A). $\mathrm{IC}_{50}$ values were determined from the TUNEL assay as described in Materials and methods (B). The values represent the mean \pm SD of 3 independent experiments. ${ }^{a} \mathrm{p}=0.002$, ${ }^{\mathrm{b}} 0.002,{ }^{\mathrm{c}} 0.0008$ and ${ }^{\mathrm{d}} 0.004$.

in a dose-dependent manner (Fig. 1A). Similar response was noted in BT474 also.

$\mathrm{IC}_{50}$ values were determined by graphical extrapolation on semi-log graphs, where we observed that at least $0.016 \mu \mathrm{M}$ of NRC-AN-019 was required for $50 \%$ apoptotic induction in BT474 cells when compared to $0.026 \mu \mathrm{M}$ of Lapatinib. In MDAMB231-treated cells, IC $_{50}$ value of NRC-AN-019 was $0.085 \mu \mathrm{M}$ compared to Lapatinib, which was $7.01 \mu \mathrm{M}$ (Fig. 1B).

$N R C-A N-019$ is more efficient in retarding the angiogenic potential of MDAMB231 and BT474 cells than Lapatinib. To determine the inhibition of angiogenic potential by NRC-AN019, MDAMB231 and BT474 cells were treated with Lapatinib (0-105.9 $\mu \mathrm{M})$ or NRC-AN-019 (0-193.25 $\mu \mathrm{M})$ followed by human dermal endothelial cells (HMEC) grown in the presence of conditioned media from these cells. We observed that under control conditions, HMEC cultured with MDAMB231 or BT474-conditioned media formed a network-like structure, which is indicative of angiogenesis. MDAMB231 cells treated with NRC-AN-019 or Lapatinib showed a dose-dependent response of angiogenic inhibition with NRC-AN-019 showing more angiogenic inhibition than Lapatinib. BT474 cells treated with Lapatinib showed little angiogenic inhibition as compared to NRC-AN-019 treatment (Fig. 2A). Quantitative analysis 

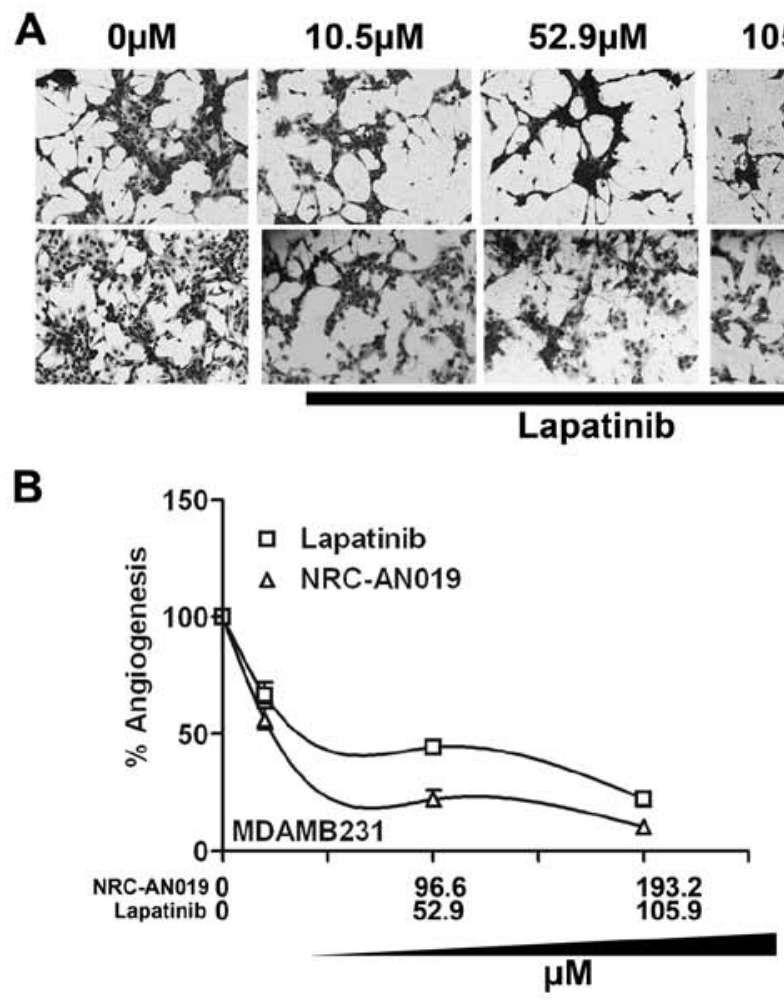
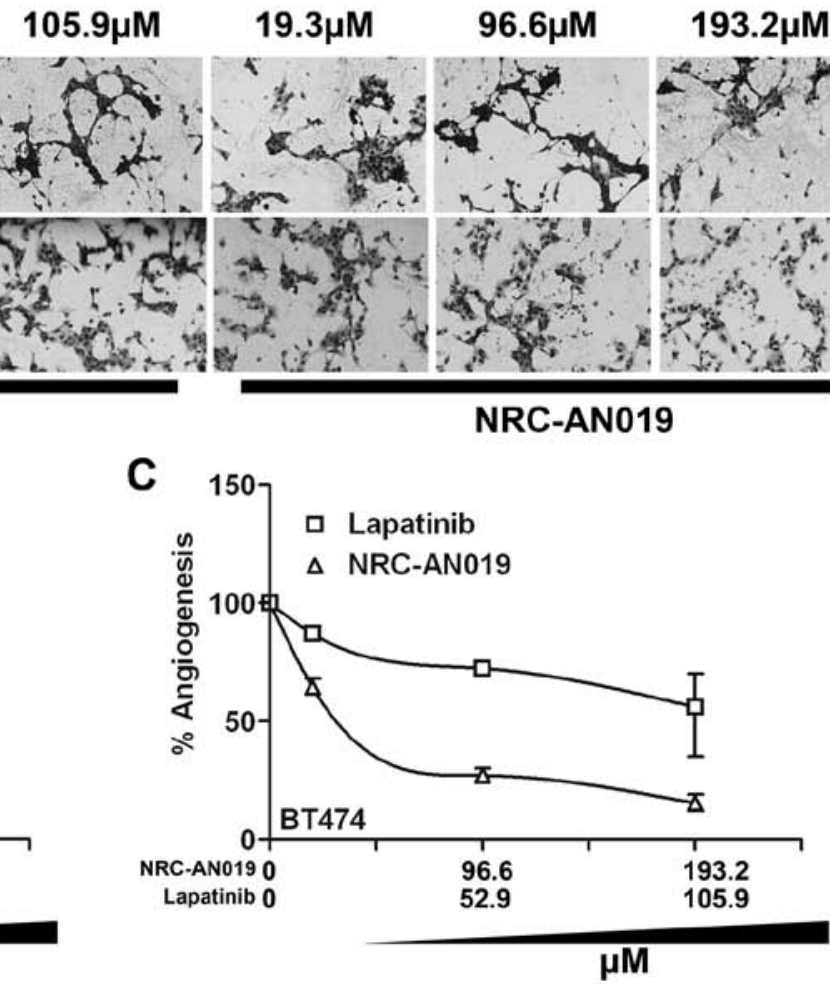

Figure 2. NRC-AN-019 causes suppression of angiogenic potential. Human breast cancer cells MDAMB231 and BT474 were treated with varying concentrations of NRC-AN-019 or Lapatinib. Conditioned media for these cells was collected and used to grow HMEC cells, which show the network-like structure formation indicative of angiogenesis (A). Quantitative analysis of angiogenesis in MDAMB231 (B) and BT474 (C) cells was performed as described in Materials and methods.

revealed that MDAMB231 cells had a similar decrease in angiogenic potential with either NRC-AN-019 or Lapatinib treatment. However, NRC-AN-019 showed better reduction in angiogenesis than Lapatinib in MBAMB231 cells at comparable concentrations (Fig. 2B). Similar reduction in angiogenic potential was also seen in BT474 cells (Fig. 2C).

$N R C-A N-019$ retards the proliferative potential of MDAMB231 and BT474 cells. We further determined the inhibition of proliferation in BT474 and MDAMB231 cells treated with various concentrations of NRC-AN-019 or Lapatinib using standard MTT assay. Quantitative analysis of percent proliferation by MDAMB231 breast cancer cells treated with various concentrations of NRC-AN-019 or Lapatinib revealed that both NRC-AN-019 and Lapatinib treatments had a dose-dependent effect in decreasing proliferation (Fig. 3A). Similarly, quantitative analysis of percent proliferation by BT474 cells treated with various concentrations of NRC-AN-019 or Lapatinib revealed that Lapatinib treatment did not result in a dose-dependent decrease in proliferation when compared to NRC-AN-019 treatment (Fig. 3B).

To confirm the decrease in proliferation in MDAMB231 and BT474 cells by NRC-AN-019, we determined the expression levels of $\mathrm{Ki}-67$ using Western blot analysis. Expression levels of Ki-67 in MDAMB231 cells decreased in a dosedependent manner in cells treated with both Lapatinib and NRC-AN-019. The decrease of Ki-67 in NRC-AN-019-treated MDAMB231 cells was more potent than in cells treated with Lapatinib at comparable concentrations. BT474 cells had the least decrease in Ki-67 expression levels after Lapatinib treatment whereas NRC-AN-019 treatment resulted in a dosedependent decrease in Ki-67 expression (Fig. 3C). GAPDH expression levels served as loading controls.

NRC-AN-019 causes the accumulation of Lapatinib-resistant BT474 cells in the G0/1 phase and induces PARP cleavage in a dose-dependent manner. To further determine the effect of NRC-AN-019 on the cell cycle, we carried out FACS analysis as per standard protocols. Dose-dependent accumulation of MDAMB231 in sub G0/1 phase was observed when treated with increasing concentrations of Lapatinib and NRC-AN-019. However, greater accumulation was seen in NRC-AN-019treated cells (Fig. 4). In contrast to MDAMB231 cells, BT474 cells behaved differently when treated with Lapatinib and exhibited no dose-dependent accumulation of cells in the sub G0/1 phase. However, like the MDAMB231 cells, BT474 cells treated with NRC-AN-019 showed a dose-dependent increase of cells in the sub G0/1 phase (Fig. 4A). Quantitative analysis revealed that NRC-AN-019 more efficiently causes accumulation of both MDAMB231 and BT474 cells in the sub G0/1 phase when compared to Lapatinib (Fig. 4B).

To further confirm the accumulation of cells in the sub G0/1 phase, Western blot analysis for cleaved PARP was carried out. We observed that both NRC-AN-019 and Lapatinib caused an increase in PARP cleavage in a dose-dependent manner in MDAMB231 cells, with NRC-AN-019 showing increased cleavage of PARP when compared to Lapatinib at comparable doses. In BT474 cells, little or no PARP cleavage was observed 


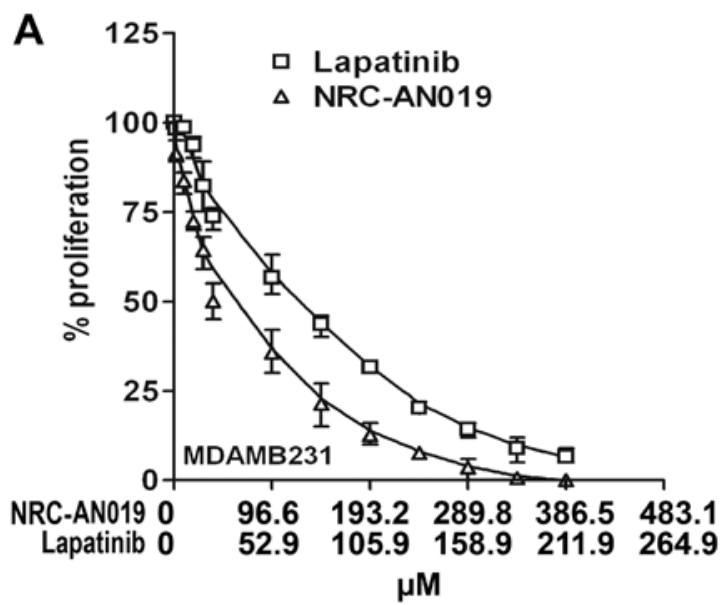

B
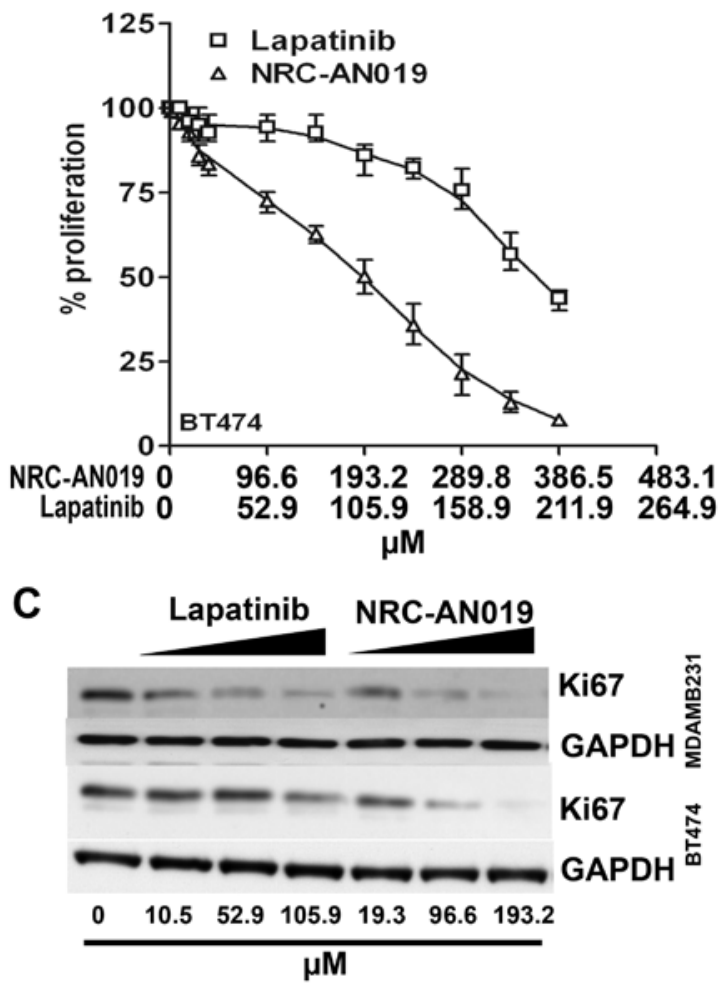

Figure 3. NRC-AN-019 decreases the proliferative potential of MDAMB231 and BT474 breast cancer cells. Cell proliferation of breast cancer cells MDAMB231 (A) or BT474 (B) cells when treated with NRC-AN-019 $(0-386.5 \mu \mathrm{M})$ or Lapatinib $(0-211.9 \mu \mathrm{M})$ was determined using MTT assay in a $96-w e l l$ plate as per standard protocols. Proliferation was quantified and graphically represented as percent of controls of MDAMB231 (A) and BT474 (B) cells. Expression levels of Ki-67 were determined by Western blot analysis of MDAMB231 and BT474 cells treated with varying concentrations of NRC-AN-019 or Lapatinib (C). GAPDH expression levels served as loading controls.

after Lapatinib treatment whereas NRC-AN-019 treatment caused a dose-dependent increase in the cleavage of PARP, which is indicative of nuclear fragmentation (Fig. 4C).

NRC-AN-019 retards the phosphorylation of EGFR in BT474 cells. To determine whether NRC-AN-019 inhibits EGFR expression or inhibits the phosphorylation of EGFR, we performed Western blot analysis for EGFR and pEGFR in BT474 and MDAMB231 cells treated with various concentrations of
NRC-AN-019 or Lapatinib. Both NRC-AN-019 and Lapatinib caused a dose-dependent decrease in expression levels of pEGFR; notably, pEGFR was undetectable in all NRC-AN019 treatments. Interestingly, expression levels of total EGFR did not change significantly after treatment with Lapatinib. In contrast, NRC-AN-019 treatment decreased expression levels of EGFR. There was no observable decrease in pEGFR or total EGFR expression in BT474 cells treated with Lapatinib. However, after NRC-AN-019 treatment, we observed a dosedependent decrease in expression levels of pEGFR in BT474 cells. Expression levels of total EGFR did not change significantly, and we observed only a slight decrease after treatment with 193.2 $\mu \mathrm{M}$ of NRC-AN-019 (Fig. 5A). Quantification of pEGFR expression by ELISA for total EGFR revealed that NRC-AN-019 at doses below $1.9 \mu \mathrm{M}$ was effective at retarding phosphorylation of EGFR in MDAMB231 cells, whereas a dose range between 9.6 and $19.3 \mu \mathrm{M}$ was necessary to achieve $50 \%$ reduction in pEGFR levels in BT474 cells. Lapatinib treatment was effective at inhibiting EGFR phosphorylation in MDAMB231 cells (21.1-26.4 $\mu \mathrm{M}$ of Lapatinib to achieve $50 \%$ inhibition of EGFR phosphorylation), whereas even high concentrations of Lapatinib $(>211.9 \mu \mathrm{M})$ were ineffective at achieving 50\% reduction of EGFR phosphorylation in BT474 cells (Fig. 5B).

NRC-AN-019 retards Lapatinib-resistant subcutaneous BT474 tumor growth in a dose-dependent manner in nude mice. To further validate whether NRC-AN-019 could have an anti-tumor effect in vivo, we subcutaneously implanted BT474 cells in SCID mice and treated the mice with 10, 20 or $40 \mathrm{mg} / \mathrm{kg}$ once daily with NRC-AN-019 or 30 or $100 \mathrm{mg} /$ $\mathrm{kg}$ of Lapatinib twice daily. We observed that in the controls, tumors developed uniformly and were of comparable size in both male and female mice. Mice treated with $10 \mathrm{mg} / \mathrm{kg}$ of NRC-AN-019 showed a decrease in tumor size when compared to the controls with no discernable variation between male and female mice. A further decrease in tumor size was observed in mice treated with $20 \mathrm{mg} / \mathrm{kg}$ of NRC-AN-019. Mice treated with $40 \mathrm{mg} / \mathrm{kg}$ of NRC-AN-019 showed the most significant decrease in tumor size with female mice showing the most decrease. Lapatinib-treated mice at $30 \mathrm{mg} / \mathrm{kg}$ were similar to controls while we observed a dose-dependent decrease in tumor size in mice treated with $100 \mathrm{mg} / \mathrm{kg}$ of Lapatinib. Of note, the decrease in tumor size in mice treated with $10 \mathrm{mg} / \mathrm{kg}$ of NRC-AN-019 was similar to mice treated with $100 \mathrm{mg} / \mathrm{kg}$ of Lapatinib (Fig. 6A). Quantification of tumor volumes revealed that mice treated with $10 \mathrm{mg} / \mathrm{kg}$ of NRC-AN-019 had a tumor volume of $4.3 \pm 0.2 \mathrm{~mm}^{3}$, mice treated with $20 \mathrm{mg} / \mathrm{kg}$ had a tumor volume of $4.1 \pm 0.1 \mathrm{~mm}^{3}$, and mice treated with $40 \mathrm{mg} / \mathrm{kg}$ had a tumor volume of $2.4 \pm 0.2 \mathrm{~mm}^{3}$. Mice treated with $30 \mathrm{mg} /$ $\mathrm{kg}$ of Lapatinib had a tumor volume of $11 \pm 1.0 \mathrm{~mm}^{3}$, and mice treated with $100 \mathrm{mg} / \mathrm{kg}$ had a tumor volume of $4.8 \pm 1.2 \mathrm{~mm}^{3}$; control animals had a tumor volume of $13.2 \pm 1.0 \mathrm{~mm}^{3}$ (Fig. 6B). $(\mathrm{p}>0.002)$

To validate the in vitro results for $\mathrm{pEGFR}$ inhibition, we carried out immunohistochemical analysis for pEGFR expression. Control tumors showed intense staining of pEGFR as observed from DAB reaction for HRP. A dose-dependent decrease in the expression levels of pEGFR was observed in tumor sections of mice treated with NRC-AN-019. Tumor 
A
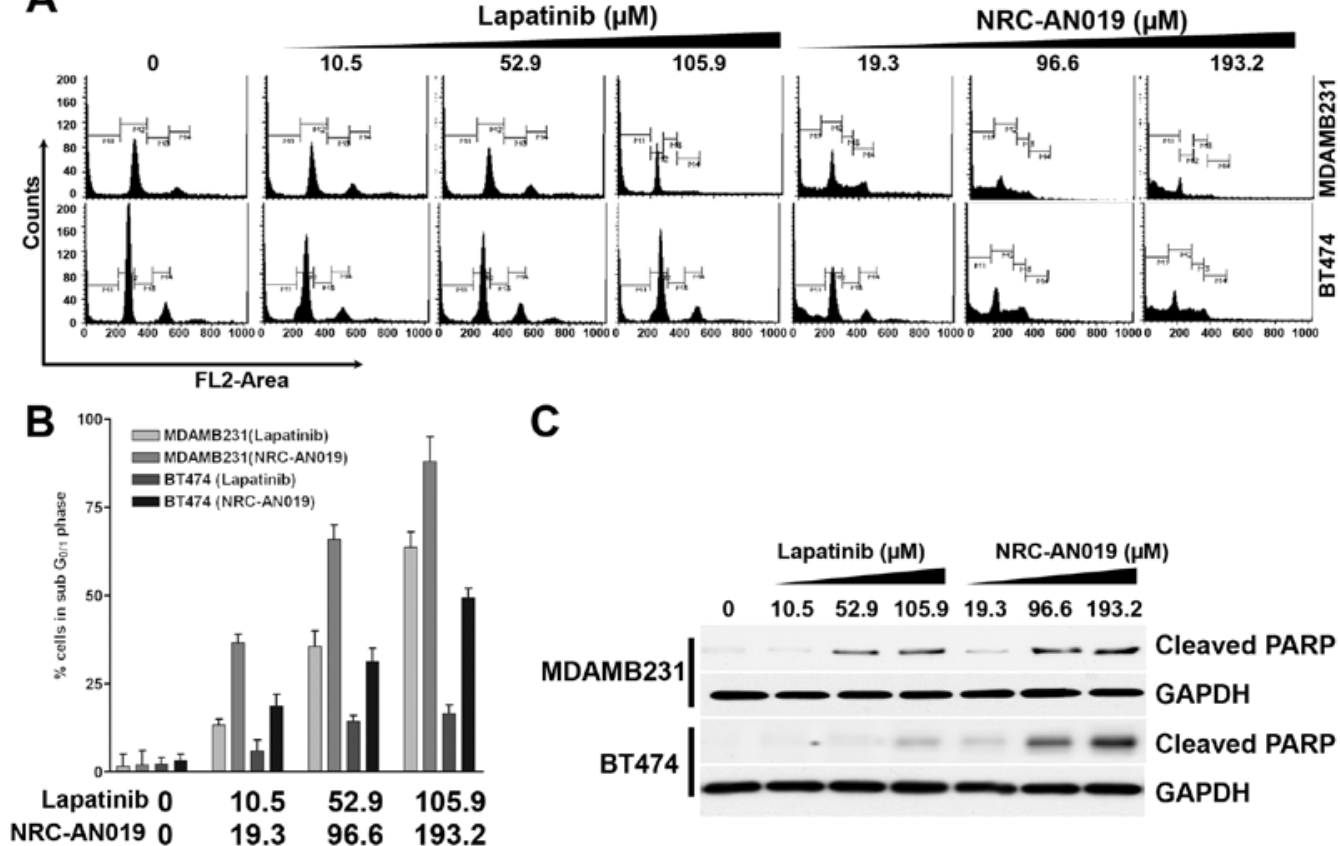

C

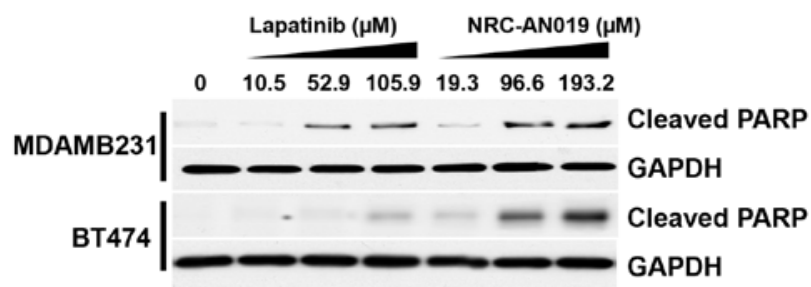

Figure 4. NRC-AN-019 treatment causes apoptotic induction in MDAMB231 and BT474 breast cancer cells. MDAMB231 and BT474 cells were treated with Lapatinib $(0-105.9 \mu \mathrm{M})$ or NRC-AN-019 $(0-193.2 \mu \mathrm{M})$ at indicated concentrations and cultured for $12 \mathrm{~h}$. Cells were then trypsinized and treated with $50 \mu \mathrm{g} / \mathrm{ml}$ propidium iodide $+0.001 \%$ RNAse-A, and sorted by standard flow cytometry (A). From the flow cytometry data, the percentage of cells in sub G0/1 were determined and are graphically presented (B). To confirm apoptotic induction, expression levels of cleaved PARP were determined by Western blot analysis of MDAMB231 and BT474 cells treated with varying concentrations of NRC-AN-019 or Lapatinib (C). GAPDH expression levels served as loading controls.

A

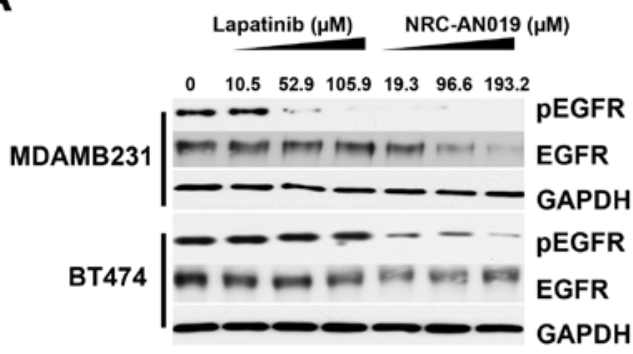

B

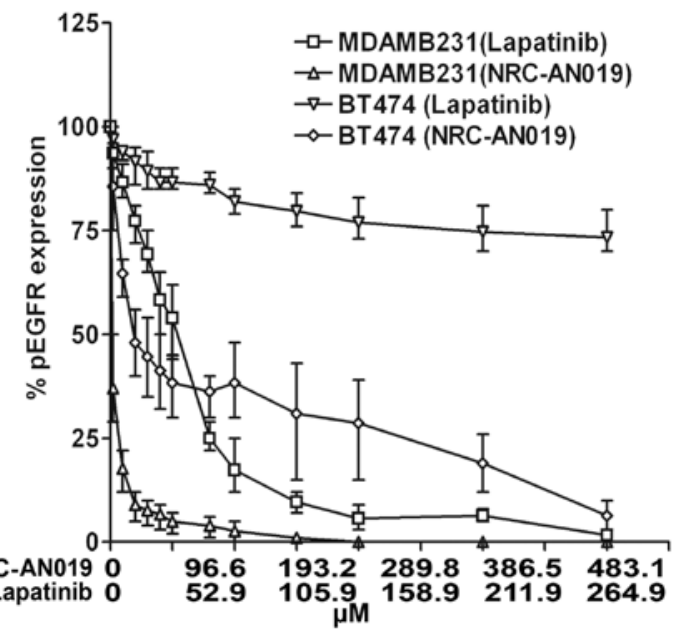

Figure 5. NRC-AN-019 inhibits EGFR phosphorylation in MDAMB231 and BT474 breast cancer cells. MDAMB231 and BT474 cells were treated with NRC-AN-019 or Lapatinib at specified concentrations. Twenty-four hours after treatment, cells were collected and cell lysates extracted. Equal quantities of proteins $(100 \mu \mathrm{g})$ were fractionated by SDS-PAGE and expression levels of pEGFR and EGFR were determined (A) with GAPDH levels serving as loading controls. Quantitative analysis of pEGFR expression in MDAMB231 and BT474 cells treated with NRC-AN-019 or Lapatinib at specified concentrations was carried out using ELISA and is graphically presented (B). sections from mice treated with Lapatinib also showed a dosedependent decrease in $\mathrm{pEGFR}$ expression but the decrease was not as significant as those observed with NRC-AN-019 treatment (Fig. 6C). Quantitative analysis of pEGFR expression normalized to controls indicated that NRC-AN-019-treated mice had a pEGFR expression of $40 \pm 11 \%(10 \mathrm{mg} / \mathrm{kg}), 22 \pm 7 \%$ $(20 \mathrm{mg} / \mathrm{kg})$ and $7.5 \pm 2 \%(40 \mathrm{mg} / \mathrm{kg})$. In contrast, mice treated with Lapatinib had a pEGFR expression of $76 \pm 5 \%(30 \mathrm{mg} / \mathrm{kg})$ and $61 \pm 2 \%(100 \mathrm{mg} / \mathrm{kg})$ (Fig. 6C).

\section{Discussion}

Preclinical experiments, clinical experience with the use of trastuzumab beyond progression, and a phase III clinical trial with Lapatinib (a dual EGFR/HER-2 tyrosine kinase inhibitor) demonstrate that the HER-2 signaling axis remains an important therapeutic target (6). Trastuzumab is a monoclonal antibody targeted against the human epidermal growth factor receptor (HER-2), which is overexpressed in $\sim 25 \%$ of invasive breast cancers. However, the majority of patients with metastatic breast cancer who initially respond to trastuzumab demonstrate disease progression within one year of treatment initiation (7).

In the present study, we demonstrate that NRC-AN-019 shows greater anti-tumor activity than Lapatinib. From our TUNEL assay results, it is evident that NRC-AN-019 shows good activity in BT474 cells even at doses as low as $19.3 \mu \mathrm{M}$ and also has a significantly low $\mathrm{IC}_{50}$ value when compared to Lapatinib.

Extensive laboratory data suggest that angiogenesis plays an essential role in breast cancer development, invasion and metastasis (8). Hyperplastic murine breast papillomas (9) and histologically normal lobules adjacent to cancerous breast tissue 
A

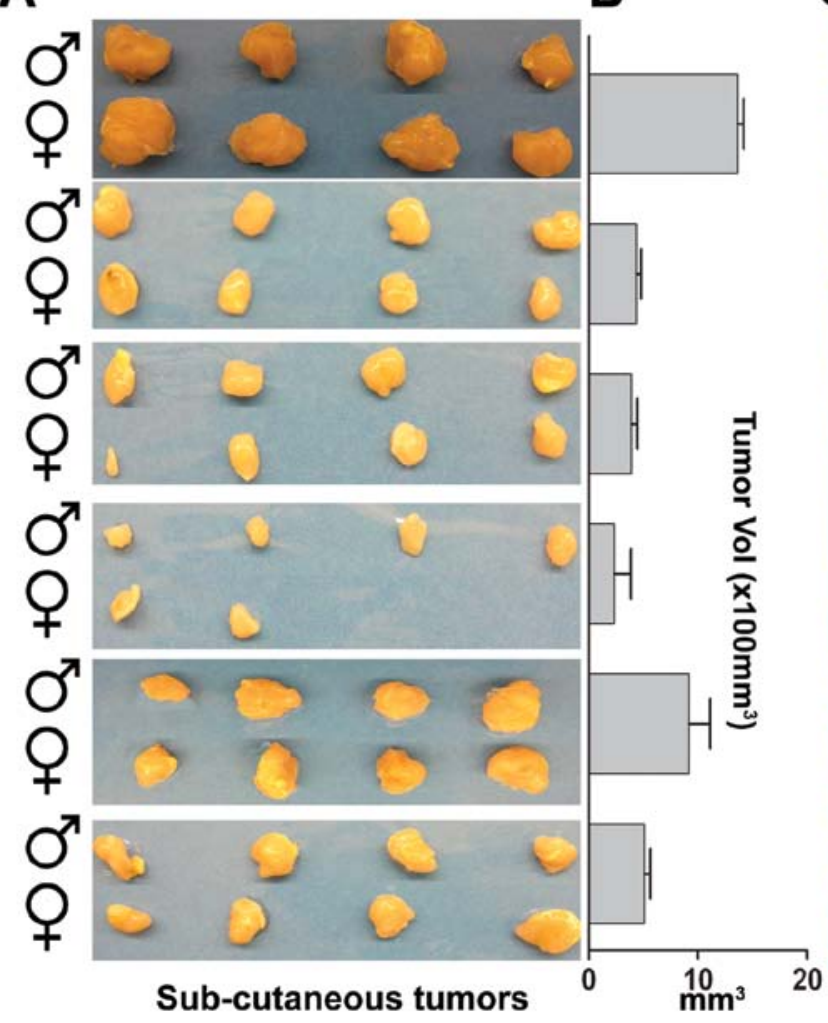

C

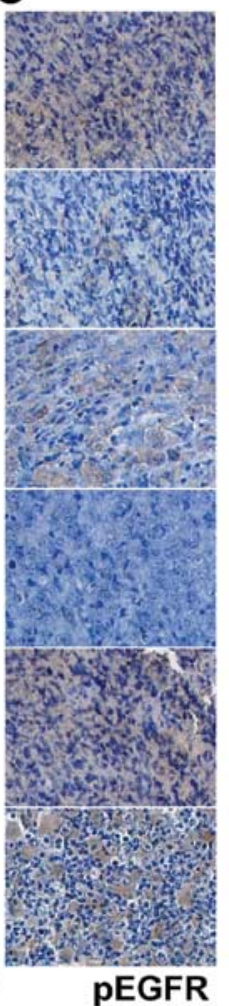

D

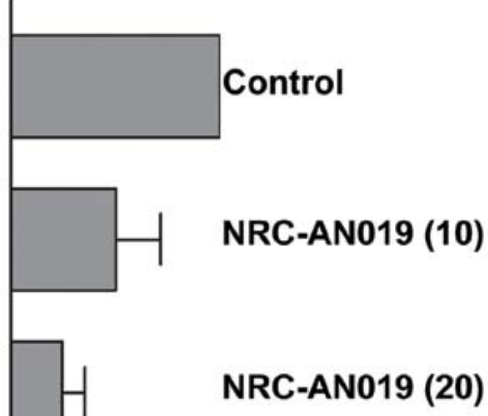

NRC-AN019 (40)

Lapatinib (30)

Lapatinib (100)

Figure 6. NRC-AN-019 inhibits subcutaneous BT474 tumor growth in SCID mice. SCID mice were subcutaneously implanted with $2 x 10^{6}$ BT474 cells. After tumors (2-3 mm diameter) developed, drug treatment was initiated as follows. Lapatinib: 30 and $100 \mathrm{mg} / \mathrm{kg}$ (two times a day) for $21 \mathrm{days}$ by oral gavage; NRC-AN-019: 10, 20 and $40 \mathrm{mg} / \mathrm{kg}$ once daily for 21 days by oral gavage; appropriate vehicle controls were included. The animals were sacrificed 30 days after the initiation of drug treatments, and tumors were harvested (A). Tumor volumes were determined as described in Materials and methods, and are graphically presented (B). Immunohistochemistry for expression levels of pEGFR was done by standard protocols using DAB HRP substrate. The expression of pEGFR was visualized as brown stain. Sections were counterstained with H\&E and photographed (C). Quantitative analysis of pEGFR expression was done by determining the DAB coloration pixel density per 10 fields using a 1024x1024 image and graphically presented as percent of expression (D).

(10) support angiogenesis in preclinical models, suggesting that angiogenesis precedes transformation of mammary hyperplasia to malignancy. Transfection of tumor cells with angiogenic stimulatory peptides has been shown to increase tumor growth, invasiveness and metastasis $(8,11-14)$. Conversely, transfection of tumor cells with inhibitors of angiogenesis decreases growth and metastasis (15).

Several strategies have been developed to target the angiogenic aspect of breast cancers but have met with little success (16). Hence, targeting angiogenesis is of critical importance at controlling metastatic breast cancers. NRC-AN-019 is a promising candidate for use as an anti-angiogenic agent. Our in vitro studies demonstrate that NRC-AN-019 has a significant anti-angiogenic effect.

In addition to significant anti-angiogenic activity, NRC-AN019 had a potent anti-proliferative effect. Although not considered an obligatory marker, Ki-67 is also frequently measured both as a static marker of proliferative activity and as a possible dynamic intermediate or surrogate marker of treatment efficacy. The expression of Ki-67 varies in intensity throughout the cell cycle, and this has raised concern that it could lead to a misclassification of cycling cells as resting ones (17). Overall, evidence indicates that levels of $\mathrm{Ki}-67$ are low during G1- and early $\mathrm{S}$-phase and progressively increase to reach a maximum during mitosis, hence making it a good indicator and the expression of $\mathrm{Ki}-67$ has often been referred to as $\mathrm{Ki}-67$ index for proliferative index (18-20).

In our study, the determination of Ki-67 levels indicates that treatment of both MDAMB231 and BT474 cells with NRC-AN-019 did decrease the expression of Ki-67 and confirms the results from the MTT proliferation assay. These results demonstrate that $\mathrm{NRC}-\mathrm{AN}-019$ has the potential to retard the proliferation of Lapatinib-resistant breast cancer cells in a dose-dependent manner and may have significant therapeutic potential as an anti-proliferative and anti-angiogenic agent. Targeting these types of cells with relevant contributing genetic modifications is necessary. Our cell cycle studies show that even at relatively low concentrations $(19.3 \mu \mathrm{M}), \mathrm{NRC}-\mathrm{AN}-019$ caused significant disruption of the cell cycle in BT474 cells. This experiment was only carried out for $12 \mathrm{~h}$; an extended experiment may produce a greater response at lower concentrations.

The apoptotic effect of NRC-AN-019 was confirmed by measuring the levels of cleaved PARP, which is indicative of DNA damage (21). The broad anti-kinase activity of NRC-AN019 was evident from ELISA and Western blot analysis for the expression of pEGFR. We observed that pEGFR levels were significantly lower in NRC-AN-019-treated cells than in Lapatinib-treated cells at comparable concentrations.

A number of EGFR inhibitors have been tested in breast cancer clinical trials but have had limited effect (22), thereby 
making the development of new chemotherapeutic agents all the more relevant. Our in vivo studies clearly demonstrate that at comparable concentrations, NRC-AN-019 is more effective than Lapatinib in retarding BT474-induced tumors. NRC-AN-019 even showed significant inhibition of pEGFR in vivo when compared to Lapatinib.

In conclusion, our study demonstrates that NRC-AN-019 has a lower $\mathrm{IC}_{50}$ than Lapatinib with strong anti-angiogenic, anti-proliferative, and pro-apoptotic properties in EGFRoverexpressing cells. The in vivo studies also demonstrated that NRC-AN-019 has a better anti-tumor activity than Lapatinib. These results suggest the therapeutic potential of NRC-AN-019 as an anticancer agent for the treatment of breast cancer.

\section{Acknowledgements}

We thank Shellee Abraham for assistance in manuscript preparation and Diana Meister and Sushma Jasti for manuscript review. This research was supported by NATCO Pharma Limited, Hyderabad, India.

\section{References}

1. van der Schouw YT: Incidence and mortality of cardiovascular disease in postmenopausal women world-wide and relevance for preventive strategies. Climacteric 12 (Suppl 1): S1-S5, 2009.

2. Ismail MF, Aly MS, Khaled HM and Mohamed HM: Detection of HER-2/neu, c-myc amplification and p53 inactivation by FISH in Egyptian patients with breast cancer. Ger Med Sci 7: Doc03, 2009.

3. Xiao Z, Ma X, Jiang Y, Zhao Z, Lai B, Liao J, Yue J and Fang X: Single-molecule study of lateral mobility of epidermal growth factor receptor 2/HER 2 on activation. J Phys Chem B 112 : 4140-4145, 2008.

4. Strecker TE, Shen Q, Zhang Y, Hill JL, Li Y, Wang C, Kim HT, Gilmer TM, Sexton KR, Hilsenbeck SG, Osborne CK and Brown PH: Effect of lapatinib on the development of estrogen receptor-negative mammary tumors in mice. J Natl Cancer Inst 101: 107-113, 2009.

5. Gondi CS, Lakka SS, Yanamandra N, Olivero WC, Dinh DH, Gujrati M, Tung CH, Weissleder R and Rao JS: Adenovirusmediated expression of antisense urokinase plasminogen activator receptor and antisense cathepsin B inhibits tumor growth, invasion, and angiogenesis in gliomas. Cancer Res 64: 4069-4077, 2004.
6. Bedard PL, de Azambuja E and Cardoso F: Beyond trastuzumab: overcoming resistance to targeted HER-2 therapy in breast cancer. Curr Cancer Drug Targets 9: 148-162, 2009.

7. Nahta R, Yu D, Hung MC, Hortobagyi GN and Esteva FJ: Mechanisms of disease: understanding resistance to HER2targeted therapy in human breast cancer. Nat Clin Pract Oncol 3: 269-280, 2006.

8. Finn RS: Targeting Src in breast cancer. Ann Oncol 19: 1379-1386, 2008.

9. Brem SS, Gullino PM and Medina D: Angiogenesis: A marker for neoplastic transformation of mammary papillary hyperplasia. Science 195: 880-882, 1977.

10. Jensen HM, Chen I, DeVault MR and Lewis AE: Angiogenesis induced by normal human breast tissue: a probable marker for precancer. Science 218: 293-295, 1982.

11. de Waal YR, Thomas CM, Oei AL, Sweep FC and Massuger LF: Secondary ovarian malignancies: frequency, origin, and characteristics. Int J Gynecol Cancer 19: 1160-1165, 2009.

12. Hayes DF, Miller K and Sledge G: Angiogenesis as targeted breast cancer therapy. Breast 16 (Suppl 2): S17-S19, 2007.

13. Jain L, Vargo CA, Danesi R, Sissung TM, Price DK, Venzon D, Venitz J and Figg WD: The role of vascular endothelial growth factor SNPs as predictive and prognostic markers for major solid tumors. Mol Cancer Ther 8: 2496-2508, 2009.

14. Vona-Davis L and Rose DP: Angiogenesis, adipokines and breast cancer. Cytokine Growth Factor Rev 20: 193-201, 2009.

15. Miller K and Sledge GW: Dimming the blood tide: Angiogenesis, antiangiogenic therapy and breast cancer. In: Breast cancer management application of clinical and translational evidence to patient care. 2nd edition. Nabholtz JM (ed). Lippincott Williams \& Wilkins, Philadelphia, pp287-308, 2003.

16. Schneider BP and Miller KD: Angiogenesis of breast cancer. J Clin Oncol 23: 1782-1790, 2005.

17. van Dierendonck JH, Keijzer R, van de Velde CJ and Cornelisse CJ: Nuclear distribution of the Ki-67 antigen during the cell cycle: comparison with growth fraction in human breast cancer cells. Cancer Res 49: 2999-3006, 1989.

18. Bacchi CE and Gown AM: Detection of cell proliferation in tissue sections. Braz J Med Biol Res 26: 677-687, 1993.

19. Meynard P: Prognostic factors in breast cancer. Ann Chir Plast Esthet 37: 623-630, 1992 (In French).

20. Racca S, Conti G, Pietribiasi F, Stramignoni D, Tampellini M, Valetto MR, Ghezzo F and Di CF: Correlation between pS2 protein positivity, steroid receptor status and other prognostic factors in breast cancer. Int J Biol Markers 10: 87-93, 1995.

21. Decker P and Muller S: Modulating poly (ADP-ribose) polymerase activity: potential for the prevention and therapy of pathogenic situations involving DNA damage and oxidative stress. Curr Pharm Biotechnol 3: 275-283, 2002.

22. O'Donovan N and Crown J: EGFR and HER-2 antagonists in breast cancer. Anticancer Res 27: 1285-1294, 2007. 